\title{
Trois images de la technique dans le projet de communication externe des PME/PMI « high tech »
}

\section{Gino Gramaccia}

\section{(2) OpenEdition Journals}

Édition électronique

URL : http://journals.openedition.org/communicationorganisation/1563

DOI : 10.4000/communicationorganisation. 1563

ISSN : 1775-3546

Éditeur

Presses universitaires de Bordeaux

\section{Édition imprimée}

Date de publication : 1 mai 1992

ISSN : 1168-5549

\section{Référence électronique}

Gino Gramaccia, «Trois images de la technique dans le projet de communication externe des PME/ PMI « high tech » », Communication et organisation [En ligne], 1 | 1992, mis en ligne le 26 mars 2012, consulté le 19 avril 2019. URL : http://journals.openedition.org/communicationorganisation/1563 DOI : 10.4000/communicationorganisation. 1563

Ce document a été généré automatiquement le 19 avril 2019

(C) Presses universitaires de Bordeaux 


\title{
Trois images de la technique dans le projet de communication externe des PME/PMI « high tech »
}

\author{
Gino Gramaccia
}

1 Les PMI/PME de haute technologie ont une réputation bien établie (c'est, en tout cas, pour l'observateur non averti, leur première image) : purs « systémiers » repliés sur leur culture technique d'experts, "temples technologiques ${ }^{1}$ jalousement gardés par un cercle d'ingénieurs, elles auraient choisi la stratégie la moins compromettante: celle du silence qui protège énigmes et secrets d'un savoir d'initiés. Rien n'est plus faux. Leurs dirigeants ont compris à quel point une telle attitude est anti-économique. Ces sociétés ont des projets de communication polymorphes pour la valorisation globale de leurs produits, de leurs technologies, de leur métier. Tantôt par simple question de survie, tantôt par passion. C'est ce qui ressort, très clairement, d'une enquête que nous avons menée auprès de huit PME « high tech » implantées dans la région du Bordelais.

2 Encore faut-il s'entendre sur le sens d'une expression très en vogue dans la presse industrielle. D'après le Boston Consulting Group, ce domaine est spécifiquement limité aux technologies dites de "rupture", par opposition aux technologies de perfectionnement («medium tech»), d'amélioration («low tech») et traditionnelles («no tech »). Les technologies de rupture constituent ce que Smail Aït-El-Hadj appelle la " galaxie des nouvelle technologies dominantes » (information, nouveaux matériaux, biotechnologies, énergie) $)^{2}$.

3 Toutes les entreprises visitées, spécialisées dans les métiers de l'Electronique, de l'Informatique industrielle, des automatismes, gravitent dans cette galaxie. Bien sûr, quand nous parlerons de « high tech", ce sera, en partie, par convention de langage : nos interlocuteurs ont parfois reconnu volontiers que leur savoir-faire hésitait entre le « high tech » et le « medium tech ». Mais ce dernier type est-il vraiment évocateur ?3

4 Le Plan PME/PMI Communication et Performance ${ }^{4}$ rappelle judicieusement que la communication globale passe par des choses aussi simples que l'accueil. Ses promoteurs 
seront rassurés : nos entreprises, de la plus petite (8 salariés) à la plus importante (plus de deux cents salariés), proposent espaces d'accueil et de dialogue (salles de réunion équipées, salons d'attente), espaces d'exposition et de démonstration. Quant à l'accueil téléphonique - cette toute première étape dans la gestion de l'excellence-, nous témoignerons ici de sa qualité : le visiteur intempestif que je fus doit beaucoup aux premières voix aimables qui ont permis de guider son enquête.

\section{L'entreprise naissante est communicante}

5 «La PME high tech est l'entreprise qui donne le plus de cheveux blancs aux banquiers », prétend Daniel Lorre (voir note 3). Il est vrai qu'elle gère à la fois la complexité et le risque : en amont, elle doit maîtriser, pour sa production, des technologies novatrices, en aval, occuper des marchés dits « de niche »; entre temps, convaincre les investisseurs de la fiabilité de ses stratégies. Ces PME sont donc, par nécessité, des systèmes largement ouverts sur leur environnement technologique, économique et financier: «...tous les créateurs en conviennent : la pérennité d'une entreprise repose d'abord sur sa capacité à s'inscrire dans un réseau: celui qui doit relier les financiers, les scientifiques et les industriels $\|^{5}$. Loin d'être un obstacle à l'écoute et au dialogue, la très forte culture technique des PME de haute technologie se nourrit au contraire d'échanges créatifs avec des partenaires d'horizons différents. Ceci est encore plus vrai pour une entreprise naissante. Tradec Systèmes ${ }^{6}$ est un parfait exemple de PME high tech dont la création et le lancement dépendent de la capacité du créateur à faire la synthèse, pour la genèse du premier prototype, de plusieurs cultures et disciplines, et à mettre en place une première organisation adaptée. Un projet d'innovation, c'est une évidence, est avant tout un projet de communication externe destiné à consolider - en particulier auprès des investisseurs - l'idée innovante à partir d'un réseau de partenaires. Sur la proposition de l'ANVAR', Jean-Louis d'Aviau de Piolant, P-D.G de Tradec, se lance dans une étude de marché dans le temps où un pool d'ingénierie, associant les compétences d'informaticiens et d'utilisateurs (des architectes, en l'occurrence) démontre la faisabilité technologique de l'idée. Une convention de recherche, passée avec l'Université, permettra de faire l'appoint technologique nécessaire à la mise en œuvre du prototype. L'objectif à court terme est de présenter le produit en salon. Il est clair, à ce propos, qu'une démonstration réussie constitue le premier moyen, très emblématique, une communication institutionnelle, la première expression concrète d'une complexité technologique maitrisée.

Dans de très petites organisations, communiquer est la condition essentielle de survie, mais les moyens sont souvent adaptés aux exigences du partenaire. C'est le cas de la société Aquiplan ${ }^{8}$ qui a fondé sa stratégie de partenariat sur un choix d'équipement susceptible de répondre aux besoins du donneur d'ordre. Le matériel (des stations et des logiciels de conception assistée par ordinateur) devient ainsi un élément fort du dialogue professionnel. En outre, parce ce que matériel est prestigieux (il s'agit de stations Apollo et de logiciels Mentor Graphics), il intervient, auprès de clients potentiels, comme un constituant également emblématique du projet de communication institutionnelle?

7 Ainsi, les images qu'émettent à l'externe les PME high tech sont autant de déclinaisons de leur identité technologique première, définie comme une compétence originelle forte. La typologie proposée ci-dessous constitue, au terme de notre enquête, un premier effort de modélisation. On distinguera donc : 
8 1. l'image technique: c'est l'image jugée la plus appropriée pour rendre compte des activités de l'entreprise centrées sur la production de systèmes intégrés, ces séquences d'intelligence abstraite (des cartes électroniques, par exemple), constituants de systèmes plus vastes intervenant dans les fonctions d'un processus ou d'un produit.

9 2. l'image de marque : l'entreprise met l'accent, dans son projet de communication, sur la marque commerciale de son produit propre. On passe du système intégré à l'objet fonctionnel, au design étudié, participant à l'ergonomie d'une tâche.

10 3. l'image mythique : dans l'image de marque, la technique, déjà, est devenue signe, fait signe. Avec l'image mythique, on évolue vers la construction d'un récit unificateur débordant, mais en même temps enracinant le métier de l'entreprise dans l'imaginaire épique d'une « success story »

\section{Examinons chaque image en détail.}

\section{L'image technique}

11 L'entreprise valorise son activité dans un discours à dominante technique. Pascale Weil parle du « discours de l'« activité » ou du métier ». Il s'agit, selon elle, d'un « discours plus " modeste ", un discours où l'entreprise s'efface derrière sa production (...), tourné vers le secteur d'activité ${ }^{10}$ L'objectif est de réaliser, à partir de différents moyens de communication adaptés (plaquette résumant le savoir-faire, journées portes ouvertes, salons spécialisés (limités à une seule journée), colloques scientifiques, communiqués de presse), la synthèse, le juste équilibre «signifié » entre l'emblème (figuré par le logo, la signature) et la culture technique de l'entreprise. L'image est ainsi donnée aux clients et aux prospects d'un savoir-faire maitrisé à l'exclusion de toute autre forme rhétorique. Socofram ${ }^{11}$, Bascoul Electronique ${ }^{12}$, Power Compact ${ }^{13}$ A Puissance $3^{14}$, et ICEurope ${ }^{15}$ ont choisi ce discours. L'exemple de A Puissance 3, entreprise spécialisée dans la mesure de paramètres physiques en milieu industriel, est particulièrement intéressant. Un plan de communication externe, très étudié, joint à la sobriété de l'expression le souci de l'exhaustivité. La documentation commerciale, destinée aux distributeurs, fournit en effet, dans une formulation concise et très accessible, l'intégralité des spécifications techniques des produits. La présentation a la rigueur de documents didactiques combinant une photo en noir et blanc du produit, une liste de spécifications rehaussée d'une couleur. L'élément de coût ( $1 \%$ du chiffre d'affaires pour l'ensemble du plan de communication) n'est pas étranger à ce parti-pris de sobriété. Mais il y a également ce choix réfléchi d'un discours technique informatif qu'on retrouve par ailleurs dans les communiqués de presse (une dizaine par an), et dans un bulletin d'informations, consacrés aux produits nouveaux, publié par la société. Adepte des salons (les grands salons d'une semaine, de taille internationale, et les expositions régionales d'une journée - moins coûteuses en immobilisation de personnel), A Puissance 3 a décidément la fibre pédagogique puisqu'elle s'est constituée en organisme de formation réservé aux clients et aux distributeurs. Cette dimension institutionnelle ajoute à la rigueur du style dans la communication externe, à cette stratégie du faire savoir voulue, selon les dirigeants, Marie-Christine Leblanc et Philippe Grand, comme l'expression de la politique qualité de l'entreprise.

12 ICEurope, laboratoire indépendant d'expertise de composants électroniques, a opté pour un plan de communication globalement identique : puisque l'objectif, en l'occurrence, est 
de convaincre une clientèle restreinte et homogène, autant faire l'économie des signes coûteux de la séduction. La plaquette, auto-éditée, sera sobre. Priorité, donc, à l'information scientifique et technique, par ailleurs diffusée et valorisée au sein de colloques scientifiques réunissant, au bas mot, entre dix et quinze exposants. La société partícipe également aux activités d'ateliers thématiques intervenant dans le cadre d'une association du type Loi 1901. L'originalité de la démarche, déjà visible chez A Puissance 3, consiste, de l'aveu même du P-D.G ${ }^{16}$, à fidéliser les relations commerciales en développant leur dimension contractuelle fondée en priorité sur une volonté de coopération technique. Il s'agit dès lors de constituer un réseau d'authentiques partenaires modifiant en profondeur les relations de sous-traitance conventionnelle pour évoluer sans doute vers ce que les promoteurs de L'Usine du futur appellent l'« entreprise étendue $»^{17}$.

Cette priorité accordée à la valorisation exclusive de la compétence technologique, s'explique, selon Daniel Lorre, par le «souci légitime, de la part de ces PME, d'obtenir avant tout la reconnaissance de leurs pairs ». En fait, la stratégie de valorisation est fonction du type de production (généralement des systèmes ou procédés intégrables) et du type de clientèle (de grands donneurs d'ordre comme l'Aérospatiale, IBM... ou des PME exprimant un besoin spécifique). Par ailleurs, le rôle amplificateur des médias fait craindre le pire : nombre de nos interlocuteurs ont déclaré redouter l'effet générateur de rumeurs d'une communication "tous azimuts" non maîtrisée (dans la presse grand public, par exemple). Aussi préfère-t-on «distiller» - terme souvent évoqué l'information dans la sphère, très homogène, de leurs relations de partenaires.

Mais sans doute faut-il voir, dans cette stratégie de communication, l'effet d'une culture qui veut associer, à la passion des systèmes complexes ${ }^{18}$, une "rhétorique blanche " dissimulant, à moindres frais (discursifs et financiers), ses effets de style - car enfin le langage est toujours là - sous une neutralité d'apparence. La technique est énoncée dans un discours qui ne dit rien d'autre que la rationalité des systèmes. Toute dérive dans le langage est interprétée comme un travail dans l'imaginaire et c'est cela, semble-t-il, qui est jugé comme dangereusement incompatible avec ce qui doit être dit, en intégralité, de l'ordre de la technique.

Pourtant, cette « excursion » dans l'imaginaire, les tenants de l'image de marque l'ont tentée. Une rhétorique explicite est essayée qui, à la manière - déjà conventionnelle, il est vrai - de la publicité, met en scène l'utilisateur. Ce qui, somme toute, représente une première étape dans la constitution d'un capital symbolique.

\section{L'image de marque}

16 La documentation commerciale de la société Satelec-Pierre Rolland ${ }^{19}$ emprunte au discours $t$ à l'imaginaire de la publicité toute la force persuasive de l'identification. La notice commerciale du produit « Suni » (appareil conçu pour l'implantologie dentaire) en est un bon exemple ${ }^{20}$. En couverture est représenté un chirurgien-dentiste ${ }^{21}$ (le professionnel utilisateur) dans l'ali: une sereine et détendue du spécialiste (il est représenté assis dans un fauteuil, les mains croisées derrière la nuque et les pieds posés sur son bureau (position suggérée) qui a décidé de faire confiance à Satelec-Pierre Rolland. Et pour office de tout texte, toujours en couverture, la «base line »: «Satelec prend soin de moi », assortie de la signature de l'entreprise surmontée d'un papillon rouge. Cette image est déclinée sur l'ensemble des notices de produits de la gamme. En page deux du document, est rapporté un témoignage-type intégrant deux thématiques : la 
sérénité et la haute technologie : «La sérénité, pour moi, c'est Suni... le contrôle de la vitesse en bout d'instrument, ainsi que l'asservissement du couple piloté par microprocesseur m'apportent beaucoup de confort». Est ainsi recréé un univers symbiotique dans lequel le nous et le vous se fondent en un personnage du «troisième type »: un utilisateur idéal, dont le propos, rapporté en style direct, vante les mérites fonctionnels des produits de l'entreprise. Une notice technique complète cette citation et assure ainsi la lisibilité d'un document stratégiquement essentiel à la promotion d'une marque conçue comme l'expression symbolique d'une réponse technologique précisément ajustée à un besoin. Selon Brigitte Bonnet-Vincent (responsable du service Communication), ce choix d'image doit prolonger, à l'externe, la très forte culture qualité de l'entreprise. Le discours d'identification, devenu classique, de la publicité, permet ainsi de construire un imaginaire euphorique qui réussit l'adéquation parfaite entre le besoin (très spécifique) et une réponse fonctionnelle simplifiée. Le produit n'est plus, comme dans l'image précédente, identifié à ses seules fonctions « désincarnées » - sans "valeur sémiologique ajoutée »-, mais devient l'élément constitutif d'une relation de confiance. Dans la stratégie Satelec, il y a du fiduciaire : ce discours de la confiance a besoin, pour se rendre explicite, du propos direct de l'utilisateur

Cette communication, se conjuguant au présent - le temps de la réponse adaptée à la demande -, s'oppose à la stratégie de "tous les temps », celle qui reconstruit le mythe d'un succès technologique. C'est notre troisième type - l'exemple de la société Com1.

\section{L'image mythique}

Chez Com $1^{22}$, plaquettes et film d'entreprise racontent la "success story" d'une entreprise de haute technologie fabriquant des modems, ces outils qui permettent d'établir une liaison télématique entre ordinateurs. Le marché est ouvert, la stratégie marketing efficace: le succès est foudroyant (soixante-quatre millions de francs de chiffre d'affaires en 1991, pour plus de trente salariés) ${ }^{23}$. Un service de communication renforce la très bonne image de l'entreprise, à l'interne et à l'externe, en développant une stratégie multimédia globale : journal d'entreprise diffusé auprès des distributeurs et des clients, plaquettes polychromes, mailing (pour l'annonce aux clients de la création de nouveaux produits), publicité dans la presse, par télécopie, par minitel, sponsoring, film d'entreprise. Une charte graphique permet d'unifier la présentation des documents. Un très luxueux salon de démonstration ouvre un espace lumineux et circulaire dans un ensemble architectural interne aux cloisons de verre. Cette rage de communiquer est à la mesure du succès de l'entreprise. La PME a mis en œuvre le jeu d'outils qui lui permettent de raconter son histoire. Elle construit les signes de son succès avec le souci évident de les intégrer en un récit intemporel, symbolique, collectif, et dont l'effet le moins inattendu pourrait bien être de lexicaliser la marque commerciale : « sous peu, on ne parlera plus de «modem» mais de «coml»: tel est le vœu du service de Communication, qui ne contredit pas, par ailleurs, le sujet du film (l'inauguration, par Com 1 d'une coupure technologique dans l'histoire des techniques de la communication). Sans doute, cette mythologie répond-elle au souci d'unifier les « croyances du groupe » (Schwebig), pour les projeter, en un tout cohérent et lisible, au regard des partenaires de l'entreprise. Chez Com1, les moments où le mythe doit être entendu comme tel (ses « occurrences») sont judicieusement définis : film, communiqués de presse, plaquettes, viennent renforcer les grands événements de la société: sortie de nouveaux produits, entrée en Bourse, signature de nouveaux contrats, création de filiales... Là encore, le mythe installe de façon 
solennelle le présent dans l'histoire. En présence évidemment, des partenaires, associés, pour la circonstance, à un travail d'unification. Au fond, le mythe s'apparente, dans la PME high-tech, à une extension, dans l'imaginaire, des solutions de simplification fonctionnelle en réponse à des problèmes technologiques complexes. Ceci se conçoit d'autant mieux que la structure du mythe - faut-il relire Lévi-Strauss? - réussit l'ajustement et l'intégration d'éléments hétérogènes (ici technologiques, économiques, organisationnels, humains) et, par suite, par la simplicité parlante de son message, donne de l'entreprise une image globale d'ouverture non équivoque, obligée, rassurante mais cependant fascinante sur un univers de solutions technologiques. Coml, c'est l'« autre dimension de la communication", dit le slogan: voilà une percée d'une concision extrême dans l'univers... cosmique de la haute technologie.

Nos entreprises ont fondé leur projet de communication externe sur une volonté de cohérence interne et, par conséquent, sur la recherche d'une identité affichée exprimant des valeurs d'unité, de compétence et de qualité.

20 L'image offerte à l'externe - en premier lieu au regard des clients mais aussi bien à celui, plus hétérogène, de la cité, sera donc le reflet de cette synergie efficace et savante, de cette personnalité industrielle dotée d'une psychologie homogène et simple, fruit de l'obsession mutualiste des managers, très ouverts aux techniques du management moderne. Cette personnalité doit combiner des qualités de motivation, de transparence, de civisme et de passion. Les projets de communication interne de Satelec-Pierre Rolland et de la société I2S vont explicitement dans ce sens.

21 Alain Ricros, P-D.G. d'I $2 \mathrm{~S}^{24}$ œuvre, sur les plans interne et externe, pour cette métamorphose civique de son entreprise, la revêtant des habits de la citoyenneté. Impliqué dans un projet éducatif extérieur à l'entreprise (la création d'un lycée privé déconventionné), il prolonge ainsi, dans la sphère civile - et nécessairement décalée par rapport à son métier - la logique d'efficacité et de performance dont se réclame l'entreprise. Dans ce cas, la valeur ajoutée n'est plus simplement le signe (qui permet de construire le mythe de la réussite), mais la vertu civique, plus abstraite et, dans cette logique, plus fédératrice ${ }^{25}$ puisqu'elle est supposée donner aux employés un sentiment de fierté. À l'interne, ce sentiment est renforcé parce qu'Alain Ricros appelle « l'organisation de la transparence ", dans le but évident de prévenir secrets, rumeurs et polémiques, mais surtout de permettre l'exercice d'un langage fédérateur - ce qui est, somme toute, l'effet premier de tout langage. Chez Satelec, cette transparence trouve son support le mieux adapté dans le journal interne («Pluri-News») qui associe, dans ses rubriques (sous la responsabilité, au sein du personnel, d'un réseau de correspondants), le discours épique du nécessaire engagement autour de quelques idées-forces (innovation, anticipation, respect du client, service et qualité, communication permanente) et le langage plus diversifié, plus composite, d'un magazine traitant de sujets généraux et d'actualité. Selon Brigitte Bonnet-Vincent, le journal, d'orientation très sociale, doit évoquer la vie de l'employé dans une visée plus emblématique que polémique. Il est pardessus toute l'expression de la politique qualité de l'entreprise. C'est le support d'un discours collectif engagé non pas dans un combat (le style de l'éditorial n'est pas guerrier) mais dans une quête économique qui transcende, par son humanisme adouci, les limites du savoir-faire de l'entreprise: « Tous nous devons nous engager à en faire (des idéesforces citées plus haut) notre culture et vos références personnelles dans nos métiers et nos services respectifs. C'est cet engagement, le mien comme le vôtre, qui fera que le monde sera bientôt notre ville » (Gilles Pierson, P-D.G). 
Reste à mesurer l'impact de ces projets d'image. La rentabilité d'une communication technique est vérifiée chez A Puissance 3 : à la suite de commandes, la participation de la société au salon Interchimie a été rentabilisée en un mois. Les sessions de formation des distributeurs, qui renforcent la relation de confiance, ne sont pas étrangères à ce succès. Cette stratégie de communication semble donc très adaptée aux produits de l'entreprise qui répondent, sans conteste, à un besoin très spécifique de mesures électroniques. Satelec-Pierre Rolland déclare, pour sa part, n'avoir, à ce jour, aucun projet précis d'évaluation. Il est vrai que dans le projet de cette entreprise, l'image choisie est neuve et l'effet de sa dimension proprement rhétorique est très difficilement mesurable $-\mathrm{du}$ moins à court terme. Plus généralement, toute une étude reste à faire qui montrerait les effets (mais aussi bien les possibles et les limites) de la séduction sémiologique dans le projet de communication technique.

C'est en tout cas l'approche affinée de besoins nouveaux, toujours débusqués, qui caractérise le mieux, selon nous, la dynamique de communication des PME de haute technologie. Toujours entre obsolescence et innovation, elles ont en même temps cette faculté d'écoute qui permet une interprétation fonctionnelle des besoins par leurs propres experts - et elles n'en manquent pas ! - dans le cadre d'une organisation et d'une culture appropriées. Tout ceci pourrait bien annoncer le passage d'une culture de l'oral à une culture du signe (de la "trace», comme disent les sémiologues) et pourquoi pas du document, de l'archive (écrite, audio-visuelle, informatique). Les managers, acteurs traditionnels de la communication orale ${ }^{26}$, redécouvrent (ou réinventent!), parce qu'ils ont maintenant le souci de relations commerciales stables, les codes et les privilèges de la mémoire. Par exemple, sous l'impulsion des gestionnaires de la qualité, une culture de l'écrit est en train de «prendre » qui traduit l'exigence d'une sauvegarde, avec le souci extrême du détail, des métiers de l'entreprise : l'écrit de la qualité (la « traçabilité »), tout en assurant la transmissibilté des savoir-faire, va permettre à coup sûr d'en renforcer la valeur contractuelle et symbolique.

\section{Haute technologie en aquitaine}

La haute technologie (dans son acception la plus large) représente, en Aquitaine, 44000 emplois qualifiés, dont $60 \%$ sont localisés en Gironde et 4000 chercheurs relevant du secteur public. Six secteurs sont représentés: l'Aéronautique et l'Espace (15000 employés), l'Informatique (7 000), l'Electronique (6 000), la Pharmacie (5 000), la fabrication de matériel électrique (6 000) et la fabrication de matériel de précision (3000). Cette présence a permis l'éclosion de PME/PMI dédiées à la haute technologie. (Ces statistiques sont fournies par le Club High Tech, créé en 1988, à l'initiative de la Chambre de Commerce et d'Industrie de Bordeaux et de neuf chefs d'entreprise, pour le développement d'un réseau de services, de formation et de financement d'aide à la création d'entreprises).

\section{Portrait-robot de la pme « high tech »:}

« Des effectifs réduits, un personnel jeune, hautement qualifié, expérimenté, des produits à haute valeur ajoutée, un chiffre d'affaires modeste, du moins au départ, une internationalisation précoce et une marge de progression appréciable, telles sont les 
principales caractéristiques de ces entreprises du troisième type » (Thierry Gironde, Objectif Aquitaine, octobre 1990, $\mathrm{n}^{\circ} 32$ ).

\section{NOTES}

1. Ces termes sont empruntes à L'Usine Nouvelle n²264, du 19 avril 1990, p.20, dans un dossier consacré aux PMI « saisies par le marketing ».

2. Smaïl Aït-El-Hadj, L'Entreprise face à la mutation technologique. Les Editions d'organisation, 1989, p.87 et seq. L'avènement du microprocesseur, par exemple (1971), inaugure une coupure radicale dans le traitement électronique de l'information.

3. Nous avons décidé de limiter notre enquête, par souci de cohérence, au domaine d'investigation déterminé par les critères suivants :

-la taille (entre 5 et 200 salariés), -l'implantation (Bordeaux et banlieue),

-l'âge (entre 1 et 10 ans $\mathrm{X}$ à l'exception de la société Bascoul Electronique

-agence commerciale à Pessac - qui a vingt ans),

-les technologies produites et utilisées (Electronique, Informatique industrielle).

Cette investigation a été complétée d'un entretien auprès de M. Daniel LORRE, secrétaire permanent de l'ADESO (Association pour le développement de l'Electronique dans le Sud-Ouest), qui édite le très précieux annuaire des entreprises d'Electronique d'Aquitaine, sans lequel nous aurions eu bien du mal a conduire notre travail. Nous adressons à tous nos interlocuteurs nos plus vifs remerciements pour la qualité de leurs propos et la chaleur de leur accueil. Entre la période de l'enquête (juillet 1991) et la date de publication du présent article (mai 1992), deux entreprises visitées (I2S et Tradec Systèmes) ont dû déposer leur bilan. La mort d'une organisation industrielle est un drame économique, évidemment. Mais au-delà, C'est la déception de l'espérance, l'échec des passions mises en jeu et des stratégies pourtant intelligentes, qui révoltent le plus.

4. Résultat d'une action concertée entre l'ANC (Agence Nouvelles Communications), l'APMID (Aide aux PMI en mutation ou en Développement), le CECA (le Centre Entreprises et Communication avancée), les Chambres de Commerce et d'Industrie d'Aquitaine et la DRIR (Direction Régionale d'Industries et de Recherche), ce plan doit encourager les PME/PMI à investir dans la communication globale (voir la Vie Economique du Sud-Ouest du 16 avril 1990, p. 21.

5. L'Usine Nouvelle, n²297, 3 janvier 1991. Dossier consacré aux « 150PMI high tech 1991 ».

6. TRADEC SYSTEMES, Léognan, près Bordeaux. Créée en 1990. Réalise une station graphique entièrement automatisée.

7. ANVAR : Agence Nationale pour la Valorisation de la Recherche.

8. AQUIPLAN, Eysines, près de Bordeaux. Bureau d'études en Electronique. Emploie 8 salariés.

9. «L'avenir de la communication corporate? Patrick d'Humières et Philippe Schwebig sont d'accord: sous-développée, elle ne peut que continuer à croître, répondant à un besoin fondamental des entreprises, notamment des PME/PMI. Celles-ci souvent victimes de la soustraitance, doivent affirmer leur propre existence en travaillant à la défense de leur identité avec un langage spécifique ». (Extrait d'un dossier de la revue Marketing Mix, n54, juillet-août 1991, consacré au marketing (p.60).

10. Pascale Weil Communication oblige ! Les Edtions d'organisation. 1991, p.91. 
11. SOCOFRAM, Talence, près Bordeaux. Créée en 1982. Emploie 17 salariés. Ingénierie en Informatique industrielle. Savoir-faire dominant: fabrication de machines spéciales pour la production (robots...).

12. BASCOUL ELECTRONIQUE, Pessac, près Bordeaux (agence commerciale) (siège social à Toulouse). Emploie au total 17 salariés. Spécialisée dans la distribution de matériel électronique.

13. POWER COMPACT, Mérignac, près Bordeaux. 49 salariés. Domaine d'activités : la conception et la fabrication de circuits électroniques

14. A PUISSANCE 3, Saucats, près Bordeaux. Emploie 12 salariés, dont 5 ingénieurs. « Une grande partie de la gamme A Puissance 3 est constituée d'appareils de sécurité intrinsèque. C'est-a-dire qu'ils peuvent, soit être installés directement en zone à risque d'explosion, soit être reliés a la zone dangereuse ». (Extrait de «Clin d'œil», bulletin trimestriel d'informations édité par l'entreprise, $\mathrm{n}^{\circ} 1$, 2ème trimestre 1991).

15. ICEurope, (Mérignac, près Bordeaux) compte 24 salariés. Cette entreprise est spécialisée en analyses de construction et de défaillance de composants.

16. Christian de Subercasaux, un ancien d'IBM.

17. «Dans le cadre du partenariat, le donneur d'ordre aide le sous-traitant à améliorer son niveau technique, c'est-à-dire à maîtriser la qualité, les délais, la mise en oeuvre de la productique, etc. Il s'agit d'une coopération technique dans une perspective de moyen et de long terme, et non de relations commerciales correspondant à des opportunités passagères. L'objectif du donneur d'ordre est de disposer d'un pôle de compétence dans un métier sur lequel il ne souhaite pas se spécialiser lui-même. Le développement de la sous-traitance (...) conduisent à la constitution de réseaux et à ce qu'on appelle "l'entreprise étendue ». L'Usine du futur. L'entreprise communicante et intégrée. Rapport du groupe de prospective présidé par Bernard Irion. La Documentation Française, juin 1990, pp.67, 68.

18. Philippe Grand est un passionné de mesures.

19. Le groupe dentaire SATELEC-PIERRE ROLLAND (Mérignac près Bordeaux.) emploie 200 salariés. Domaine d'activités, pour Satelec: l'Electronique dentaire. Pour Pierre Rolland: les produits de laboratoire (anesthésiques dentaires...). Part du chiffre d'affaires affectée à la communication : $2 \%$.

20. Plaquette réalisée par une agence bordelaise à partir d'un cahier des charges proposé par Brigitte Bonnet-Vincent, responsable du service de communication de l'entreprise.

21. Joué par un salarié de l'entreprise.

22. COM1. Cestas Créée en 1987. Emploie 33 salariés. Principale production: des modems miniaturisés.

23. Prévision CA $9290 \mathrm{MF}$ dont 30\% à l'export. L'entreprise a obtenu d'importants contrats auprès de Toshiba et d'IBM. Le constructeur américain lui a demandé d'équiper tous ses nouveaux ordinateurs PS1 livrés en France d'un modem permettant l'accès direct au minitel (Source Aquitaine Eco 91 - Publication du journal Sud-Ouest, p. 155).

24. I2S, Bordeaux. Emploie 43 salariés. Principale production : caméras industrielles.

25. On lira, sur ces considérations, le chapitre intitulé «L'entreprise citoyenne dans le numéro 100 (septembre 1988) de la revue Autrement, pp. 200-203. Françoise Piotet, dans Projet 221 (printemps 1990), soutient que la notion de projet d'entreprise n'est pas aussi simple. Il y aurait, dans l'entreprise, autant d'objectifs que d'acteurs: «les actionnaires souhaitent obtenir un retour maximum de leurs investissements, les cadres veulent une carrière, du pouvoir, un statut les ouvriers ou les employés un salaire qui leur permette de vivre et d'élever leurs enfants. Pour paraphraser Michel Crozier, à l'intérieur de l'entreprise, chacun poursuit ses propres buts »; (p.39).

26. Cf. Henry Mintzberg, Le Management - Voyage au coeur des organisations. Les Editions d'Organisation, 1990, p. 29 : «Les managers favorisent totalement les moyens de communication verbaux, c'est-à-dire le téléphone et les réunions ». 


\section{RÉSUMÉS}

Les PME/PMI de haute technologie ("high tech», en termes plus médiatiques) ont, dès leur création, deux projets : celui d'innover dans les sphères les plus complexes et les plus incertaines des technologies dites de "pointe", et celui de communiquer avec tous les partenaires qui accompagnent, en amont et en aval, leur création (universitaires, organismes d'aide, investisseurs et clients). Trois images de la technique sont émises, à l'externe, pour promouvoir leur projet d'entreprise: l'image technique, reflétant les compétences, l'image de marque, valorisant la marque commerciale, l'image mythique, ou image globale, qui illustre le succès de l'entreprise. C'est ce que révèle une enquête menée auprès de PME/PMI high-tech du Bordelais, spécialisées dans le traitement électronique de l'information.

\section{AUTEUR}

\section{GINO GRAMACCIA}

Gino Gramacia est maître de conférences à l'Institut Universitaire de Technologie « A » de Bordeaux. Il enseigne la Communication et la Gestion de Projet dans le Département de Génie Electrique et d'Informatique Industrielle. Il travaille actuellement sur le projet de communication externe des PME/PMI en direction des partenaires universitaires (dernière publication à paraître : «Vivre les relations École/Entreprise : une expérience du produire »-ÉTUDES été 92). 